\title{
Data-based Detection and Diagnosis of Faults in Linear Actuators
}

\author{
Cristobal Ruiz-Carcel, Andrew Starr
}

\begin{abstract}
Modern industrial facilities, as well as vehicles and many other assets, are becoming highly automated and instrumented. As a consequence, actuators are required to perform a wide variety of tasks, often for linear motion. However, the use of tools to monitor the condition of linear actuators is not widely extended in industrial applications. This paper presents a data-based method to monitor linear electro-mechanical actuators. The proposed algorithm makes use of features extracted from electric current and position measurements, typically available from the controller, to detect and diagnose mechanical faults. The features are selected to characterise the system dynamics during transient and steady-state operation and are then combined to produce a condition indicator. The main advantages of this approach are its independence from a need for a physical model or additional sensors. The capabilities of the method are assessed using a novel experimental linear actuator test rig specially designed to recreate fault scenarios under different operating conditions.
\end{abstract}

Index Terms - Fault detection, diagnosis, linear actuator, EMA, multivariate analysis

\section{INTRODUCTION}

$\mathrm{L}$ INEAR machines are all around us, covering a wide range of applications such as aircraft undercarriage and control surfaces, automatic doors, railway track point and level crossing actuators, robot arms, CNC machine tools, and many more. A failure in these actuators can be critical for the safety, performance and availability of the system in which they are integrated. In some cases, such as the operation of control surfaces in aircrafts, a fault in an actuator can also compromise the safety of passengers and crew. That was the case of the Alaska Airlines 261 flight accident in 2000, where an excess of wear in the jackscrew actuator that controls the horizontal stabilizer of the plane caused the crash of the aircraft, and the death of 88 people [1]. This clearly demonstrates the importance of monitoring the health of linear actuators.

Many different types of linear actuators are available commercially. Historically mechanical arrangements such as cam-follower or crank-rod mechanisms have been used to produce a repetitive and accurate linear motion profile. Hydraulic and pneumatic actuators have also been extensively used for applications that require the user to control the motion and/or produce high forces. More recently, electro-mechanical actuators based on the combination of an electric motor and a screw, belt and pulley, or rack and pinion mechanisms have gained popularity due to their automation and control capabilities, in addition to other benefits such as simplicity or weight reduction. The failure modes and degradation in this type of devices has not been studied in depth yet, but a comprehensive list of typical failure modes and a criticality analysis for ball-screw mechanisms was provided in [2]. Structural failures such as excess of wear, cracking, backlash or spalling, malfunctions such as ball return channel jamming or seizure and lubricant related faults such as run-dry or contamination are amongst the most severe and common mechanical faults in this type of mechanism.

Despite the increasing utilization of linear actuators and the critical role played in some of their applications, the presence of condition monitoring tools for these devices is quite limited in industrial applications and academic research. This fact can be attributed to several different causes:

- The use of condition monitoring tools is typically limited to high value assets;

- there is a large number of different typologies of linear actuators, leading to the need for specific tools for each type;

- The different assemblies use components of different nature and often require specialist knowledge about mechanical, electrical and control interfaces, creating challenges to assemble the skill set;

- Complex dynamics including nonlinearities, changing operational conditions, dominance of friction phenomena, and non-stationary operation create a challenging starting point for the design of monitoring tools.

The presence of sensors in linear actuators is typically limited to system control purposes, which is also a constraint for the implementation of condition monitoring strategies.

Despite all these difficulties, some research work has been done recently in industry and academia, particularly for linear electro-mechanical actuators (EMA). The potential application of EMAs in the more- and all-electric aircraft for operation of 
control surfaces and the landing gear [3], and the monitoring systems required, are probably the main research topics [4]. For this application, as the actuator is installed in an aircraft, the reliability of any new system needs to be auditable and related to known data [5], and be at least as good as conventional hydraulic systems. In particular, the ball-screw actuator is the most popular mechanism studied. It has been tested in several simulation and experimental studies, including its installation in a real aircraft for comparison with traditional hydraulic actuators [6]. The monitoring approaches developed in the past normally relied on mathematical models of the actuator [7][15] based on first principles. Despite the success of these approaches, the need of unique models limits its application to a specific machine and requires a specialist modeller. Other approaches used measurements of signals that normally are not available in industrial applications such as temperature or vibration [16]-[18], requiring the installation of additional sensors and wiring.

This paper presents a monitoring technique that can be applied to different types of EMA, making use of signals available from the controller or the motor drive to detect mechanical faults. Electric faults in motors is a widely studied subject [19] and it is not covered here. This research explores a monitoring method based on the automated extraction and analysis of signal features such as steady value, overshoot, settling time or presence of unexpected peaks. These features characterise the dynamic behaviour of the system. Consequently, any significant change in the condition of the system will have an effect over these signal features. Principal Component Analysis (PCA) is used to reduce the dimensionality of the extracted feature set and detect any deviations from normal behaviour. The independence from a need for a physical model, the low computation time, and the use of current and position measurements exclusively are the main advantages of this approach.

The capabilities of the proposed method are tested and assessed using experimental data. A special ball-screw test rig was designed to recreate fault scenarios under different operating conditions, such as different motion profiles, varying speeds or different loadings. This rig is also capable of a rack and pinion arrangement, not further considered here. During the experiments, different motion profiles and loading conditions were tested to evaluate the performance of the monitoring algorithm under various operating conditions. The raw data used in this investigation is available to download at [20].

If your paper is intended for a conference, please contact your conference editor concerning acceptable word processor formats for your particular conference.

\section{Methodology}

Mechanical faults such as those mentioned in the previous section may affect the operation of the mechanism and degrade its performance. If this degradation is considerable, it may have an impact in the actuator behaviour which potentially can be observed in variables measured such as the current being fed to the motor or the motion profile. Nevertheless, for this particular application the mechanism motion is not stationary, as it accelerates and decelerates in every movement repetition from one position to another. Additionally different motion profiles and different loading conditions may produce different measured values even in absence of faults, which complicates the monitoring task. However, there are certain features in those signals that are defined by the dynamic behaviour of the system and the controller, such as steady values during steady-state operation, overshoots and subsequent oscillations after a change in the position set point, etc. A change in these features may be indicative of changes in the system behaviour, which can be related to faults or malfunctions. An initial study of the main features observed in current and position measurements measured in EMA based on computational simulations was presented in [21].

The monitoring approach presented in this paper is based on the analysis of the correlation between different characteristic features extracted from current and position measurements using PCA. The objective of that analysis is to define the normal dynamic behaviour of the system by analysing key signal features under different operating conditions in absence of faults, and then look for deviations from that normal behaviour in order to detect and diagnose faults. The application of this approach consists of two major steps: first the extraction of key features from the signals that define the dynamic behaviour of the system, and second the correlation analysis using PCA.

\section{A. Feature extraction}

Fig. 1 shows an example of ideal (noise free) position measurements for a "trapezoidal motion profile" moving at constant speed to a stroke of $50 \mathrm{~mm}$, before returning to its starting point. The set point illustrated is very close to the actual position. A zoomed version of the position error, calculated as the difference between the actual position measurement and the desired set point, is represented in green. This figure shows that the actuator is able to follow accurately the desired motion profile. However, the zoomed position error signal reveals some interesting features in each of the motion sections. A steady value (a) close to zero is achieved during the major part of the movement. This movement starts with an overshoot (b) and some oscillations in the signal during the settling time (c) before the steady value is reached. In addition, unexpected

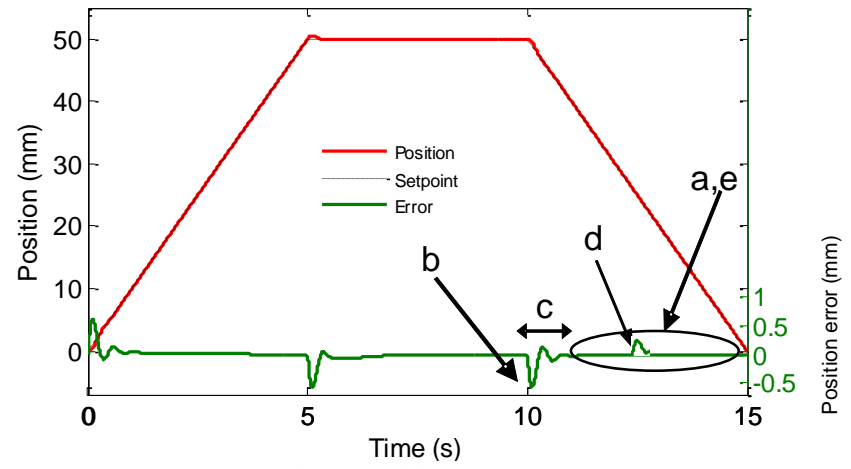

Fig. 1: Position measurements 
peaks (d) may also be visible in the steady region due to noise, external disturbances or malfunctions in the system. Finally the standard deviation (Std) during the steady region (e) can also be extracted. These features have been highlighted in Fig. 1 only for the second stage of the movement (retraction), but the same features were extracted also for the first movement (extension).

The characteristics of the current signal (Fig. 2) are very similar to the position error, and the same features can be extracted from the extension and retraction movement sections. Features $\mathrm{a}, \mathrm{b}$ and $\mathrm{c}$ are characteristic of any underdamped second order system, and it is easy to extract them for each movement (extension and retraction) and use them to monitor the condition of the system. The amplitude of unexpected peaks in the steady region (feature d) may also be indicative of faults located at a particular point in one of the contact surfaces. These features have been carefully selected to capture the dynamic behaviour of the system as well as to increase the sensitivity of the algorithm to certain types of faults. In this investigation the five features described will be extracted separately for the extension and retraction movements in the two signals analysed, position error and current.

\section{B. Principal Component Analysis of selected features}

PCA can characterize the state of a process by projecting the acquired data into a lower-dimensional space. This method has been used in many previous research works to decrease data redundancy [22] and select the most relevant features [23]. This dimensionality reduction technique preserves the correlation between the measurements capturing the data variability in an optimal way [24]. Given an $n \times m$ data set matrix $X$ containing $n$ observations and $m$ variables it is possible to obtain a set of loading vectors $V$ by solving the eigenvalue decomposition of the covariance matrix $S$ :

$$
S=\frac{1}{n-1} X^{T} X=V \Lambda V^{T}
$$

where the loading vectors $V$ are ordered by the amount of variance expressed by the corresponding eigenvalue in the diagonal matrix $\Lambda$. The loading vectors attached to the $a$ largest singular values are retained in the loading matrix $R \in \mathbb{R}^{m \times a}$. This set of vectors generates a lower dimensional representation

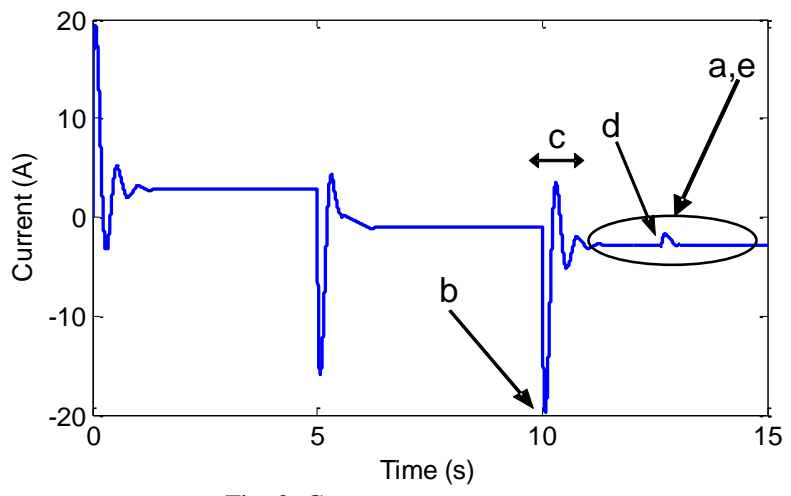

Fig. 2: Current measurements of the extracted features. The projection of the data into this reduced space captures systematic trends of the data, reducing the amount of random noise [24] minimising the negative effects of measurement inaccuracies. The score matrix $W$ contains the projection of the observed data into the lowerdimensional space, while the residual matrix $E$ represents the difference between the observations and the projection of $W$ back into the $m$-dimensional space:

$$
\begin{aligned}
& W=X R \\
& E=X-W R^{T}
\end{aligned}
$$

The indicators most commonly used for the detection of faults are the Hotelling $T^{2}$ indicator (which represents major variations in the retained space) and the squared prediction error $Q$ (representing variations in the residual space) [24]. These indicators can be computed for each observation $x$ as follows:

$$
\begin{aligned}
& T^{2}=y^{T} y \\
& Q=x^{T}\left(I-R R^{T}\right) x
\end{aligned}
$$

where $I$ is the identity matrix and the principal components $y=\Lambda_{a}{ }^{1 / 2} W^{T} x$ have been re-scaled to have unit variance [24].

The upper control limits (UCL) for $T^{2}$ and $Q$ can be calculated for a given significance level $\alpha$ such that $P\left(T^{2}<T^{2} U C L(\alpha)\right)=\alpha$ and $P\left(Q<Q_{U C L}(\alpha)\right)=\alpha$ respectively. These control limits are estimated during the training phase using data acquired in absence of faults. Later on, in the monitoring phase, the values of $T^{2}$ and $Q$ obtained are compared with these UCL to differentiate between normal and faulty behaviour. Usually these control limits are calculated assuming that the measurements are normally distributed. However, this is not the case for nonlinear systems, which makes this assumption invalid. Odiowei and Cao [25] developed a methodology to solve this issue by estimating the actual probability density function of the statistical indicators using Kernel Density Estimations (KDE). The effectiveness of this approach has also been tested using experimental data [26]. The probability of a random variable $x$ (with a probability density function $p(x)$ ) to be smaller than a certain value $s$ is defined as:

$$
P(x<s)=\int_{-\infty}^{s} p(x) d x
$$

where $p(x)$ can be calculated through the kernel function $K$ with argument $g$ :

$$
\begin{aligned}
& p(x)=\frac{1}{n h} \sum_{k=1}^{n} K\left(\frac{x-x_{k}}{h}\right) \\
& K(g)=e^{-g^{2} / 2 / \sqrt{2 \pi}}
\end{aligned}
$$

where $h$ is the selected bandwidth (see [25]) and $x_{k}$ is the $k^{\text {th }}$ sample of $x$. By replacing $x_{k}$ with $T_{k}^{2}$ and $Q_{k}$ obtained from (4) 
and (5) respectively, it is possible to estimate the probability density function of $T^{2}$ and $Q$. The respective control limits for these statistics correspond to $s$ in (6), obtained by solving $P(x<s)=\alpha$. The value of the parameter $\alpha$ is critical for the diagnosis performance, and it is chosen by the user to balance the detection sensitivity and false alarm rate.

\section{EXPERIMENTAL SET UP}

\section{A. Description of the rig}

An instrumented test rig was designed and built to evaluate the performance of the proposed algorithm. A ball-screw mechanism where threaded shaft provides a helical raceway for ball bearings housed inside a nut was selected. This arrangement allows the transformation of shaft rotation into nut linear displacement with little friction and high precision. Different typical faults were seeded in this rig under different loading conditions in order to study how these faults affect the measurements acquired from the system and how these signals can be processed for fault detection and diagnosis.

In order to simulate varying loading scenarios that represent realistic operating conditions, a second actuator was used. Both actuators were connected through a load cell, and the load measurements provided were fed back to the controller of the second actuator. Using this configuration it is possible to send a load set point command to the second actuator and take control of the external load in the actuator being tested. However, loading measurements were not used for monitoring, as in real applications they are rarely available. The proposed monitoring system must be able to cope with varying loads without using load measurements.

The test rig was designed using components available in the market for the construction of small CNC machines such as ball-screw arrangements, linear guides and bearings, stepper motors, drives, etc. Ball-screws with fitted anti backlash ballnut model RM1605-C7 with $5 \mathrm{~mm}$ lead and Nema 34 stepper motors with $4.6 \mathrm{Nm}$ holding torque were selected. The mechanical components were mounted on an aluminium profile structure. The motors of both actuators were controlled from a Labview interface, which was also responsible for the data collection.

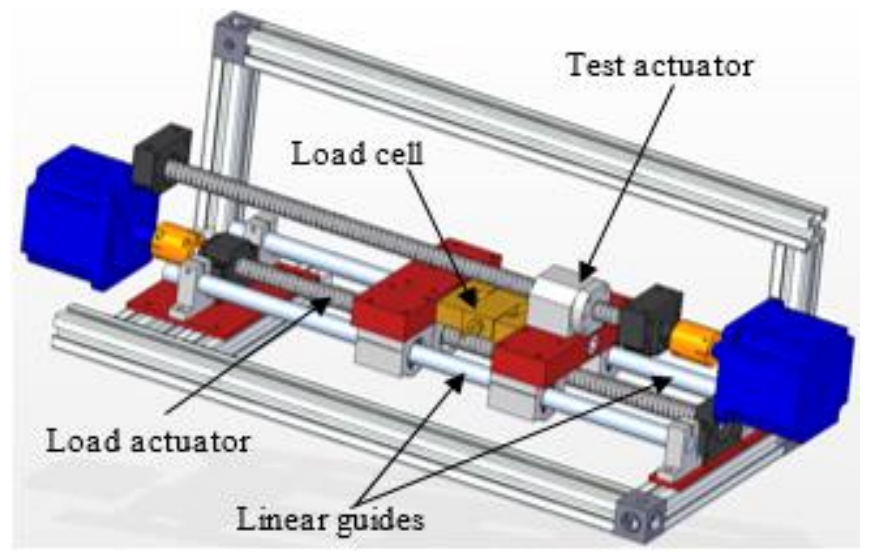

Fig. 3: 3D model of the test-rig

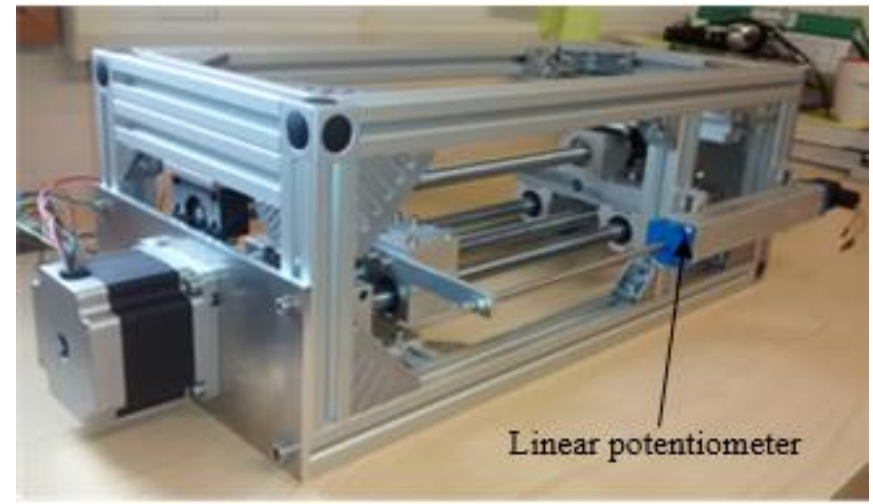

Fig. 4: Lateral view of the rig

The rig was instrumented with a series of sensors for control and monitoring purposes. For the fault detection to be effective, the sensors used need to be accurate enough in terms of resolution, bandwidth and repeatability to capture the features introduced in II.A adequately. Position was measured with a Vishay REC $115 \mathrm{~L}$ linear potentiometer, which has a linearity error of $\pm 0.025 \%$ and repeatability of $\pm 0.01 \%$. Current from the drive is measured by a Honeywell CSLA2CD Hall effect sensor with a nominal sensitivity of $32.7 \mathrm{mV}$ per cable turn $(10$ turns). In addition, for further research, nut and motor temperatures were measured using K-type thermocouples, and vibration in the nut was measured by a Dytran 3055D2 accelerometer. The load between both actuators was measured using a Tedea Huntleigh, S beam type model 614 load cell (total error $0.02 \%$ ). Load measurements were used only for controlling the load provided by the second actuator, but never for monitoring purposes. All the data was acquired at $25 \mathrm{~Hz}$, except for vibration measurements acquired at $50 \mathrm{kHz}$. The sampling rate selection was based upon a preliminary analysis of the signals acquired at a much higher frequency $(500 \mathrm{~Hz})$, where it was observed that the dynamic features described in II.A are relatively slow and can be captured accurately at $25 \mathrm{~Hz}$.

This rig design is capable of producing a displacement of 120 $\mathrm{mm}$ with a maximum external load of $392.3 \mathrm{~N}$ in both directions. The approximate dimensions of the complete rig are $0.75 \mathrm{~m}$ long, $0.35 \mathrm{~m}$ wide and $0.25 \mathrm{~m}$ high. Fig. 3 shows a CAD model of the test rig where some parts of the structure have been removed for more clarity. The nut of the actuator being tested is connected through a machined aluminium block to two linear bearings mounted on rails to avoid rotation of the nut. Pictures of the assembled test rig can be seen in Fig. 4.

\section{B. Cases studied}

Initially the rig was run under normal operating conditions (absence of faults) in order to collect a significant amount of data that can represent the behaviour of the system under different loading conditions and motion profiles. Then different faults were introduced in different parts of the system, and the signals collected were used to assess the monitoring technique and study the degradation of the actuator. The faults introduced are selected to be representative of critical failure modes of this type of machine [2].The faults tested were: 
- lack of lubrication, simulated by removing gradually the screw lubricant and tightening the nut seals (Fig. 5);

- $\quad$ spalling, simulated by artificially inducing surface defects of different sizes in the screw and balls (Fig. 6)

- backlash, simulated by replacing the original balls with balls of smaller diameter (Fig. 7).

Seeded faults were introduced because there was insufficient test time to run the ball screws naturally to failure. The faults were introduced gradually in order to observe how the severity of the faults affects the features extracted from the signals and assess the detection capabilities of the algorithm. In the case of lack of lubrication, in the first stage the lubricant was removed with degreaser. No dramatic changes were observed in the signals, mainly due to the inherent low friction of the ball-screw architecture. In order to increase the severity of the fault, the bolts holding the plastic seal at both ends of the nut containing the balls were tightened, to create more friction (see Fig. 5). The spalling defect was started as a $1 \mathrm{~mm}$ diameter surface defect on the rolling surface of the screw (see Fig. 6). In the following $2^{\text {nd }}, 3^{\text {rd }}$ and $4^{\text {th }}$ stages the size of the defect was gradually increased to 2,3 and $4 \mathrm{~mm}$ in diameter, affecting not only the bottom of the channel but also one of the sides. In stage 5 the 4 $\mathrm{mm}$ defect was replicated in a neighbour channel, and in stage 6 the size of both defects was increased affecting the sidewall between them. In stage 7 another $4 \mathrm{~mm}$ defect was seeded on the other neighbour channel of the original defect. Finally in stage 8 part of the sidewall between two of the defects was
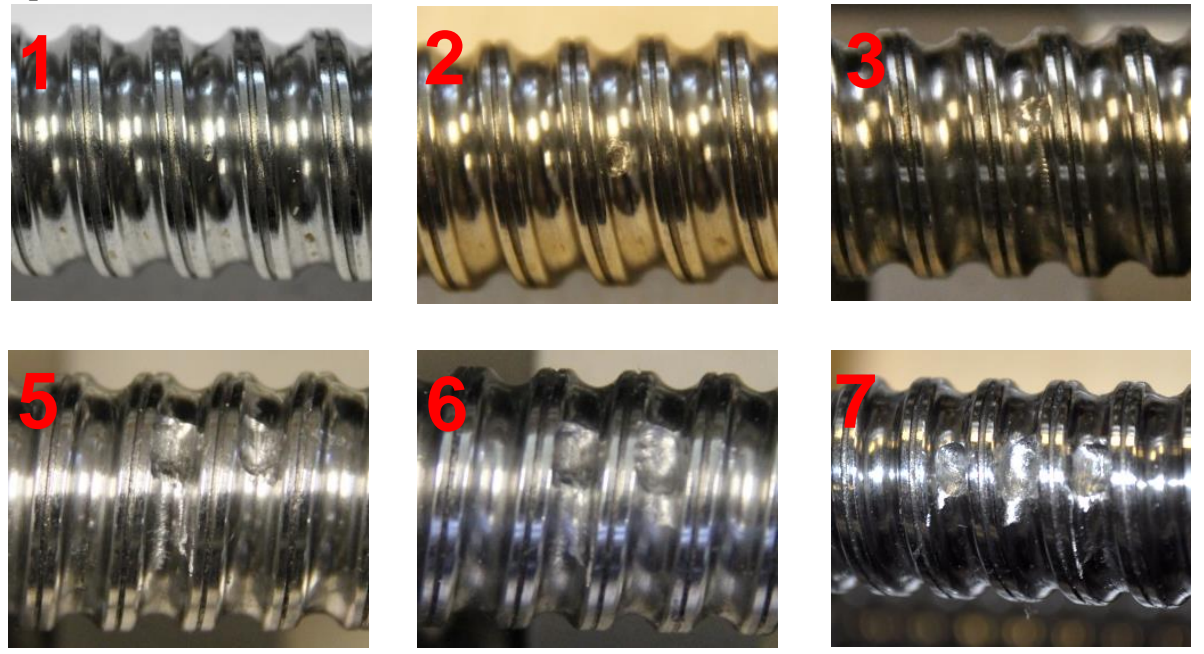

Fig. 6: 8 stages of the spalling defect

$\varnothing 3.15 \mathrm{~mm}$

$\varnothing 3 \mathrm{~mm}$

$\varnothing 2.5 \mathrm{~mm}$
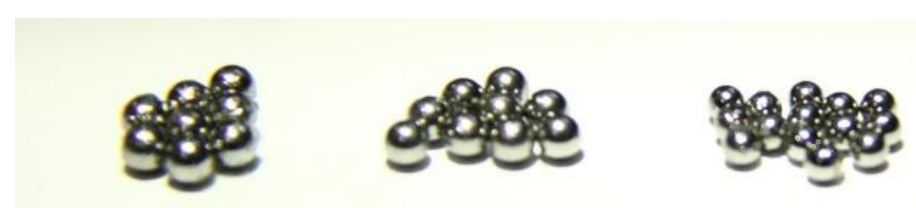

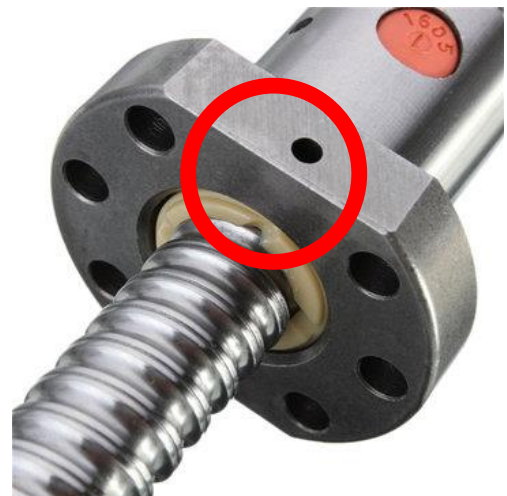

Fig. 5: Bolt holding seal in ballnut

partially removed (see Fig. 6). Backlash or excess of play was simulated replacing the original $3.15 \mathrm{~mm}$ diameter balls by 3 $\mathrm{mm}$ and $2.5 \mathrm{~mm}$ diameter balls (see Fig. 7).

The tests were performed for different motion profiles and loading conditions in order to assess the capabilities of the algorithm under different scenarios. Two motion profiles were tested. The first was a trapezoidal motion profile (constant speed set point) where the $120 \mathrm{~mm}$ stroke was completed in $5 \mathrm{~s}$ with $3 \mathrm{~s}$ waiting at both ends of the movement. The second was a sinusoidal motion profile (smooth speed transition) with $120 \mathrm{~mm}$ stroke completed in $6 \mathrm{~s}$ with $2 \mathrm{~s}$ waiting at both ends. These two motion profiles were tested for normal and faulty conditions under three different loading scenarios: $196.13 \mathrm{~N}$, $392.3 \mathrm{~N}$ and $-392.3 \mathrm{~N}$. The full motion sequence was repeated 5 times in each test, and each test was repeated 10 times in order to generate a dataset with a significant amount of observation
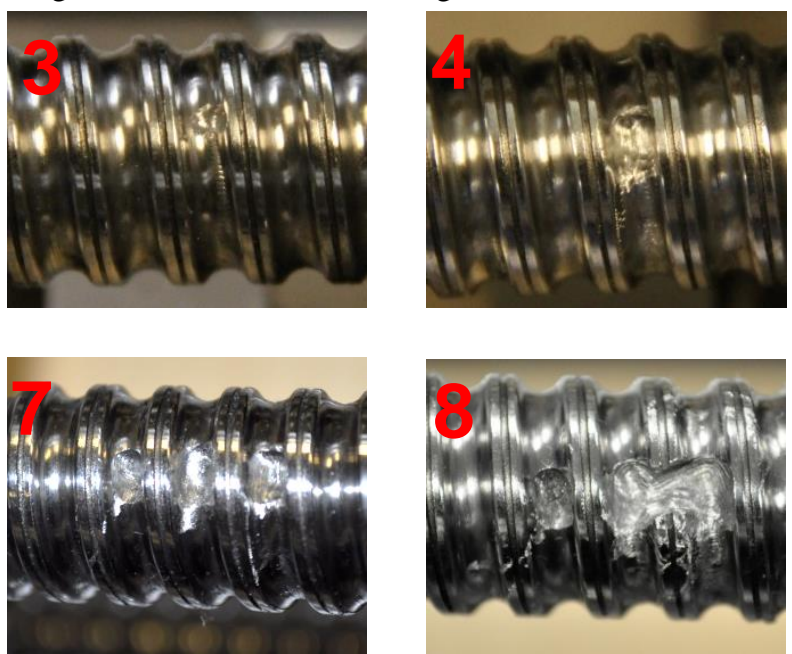

Fig. 7: Balls for simulation of backlash 
in each case studied. In every test, before the data collection started, the rig was run for about 30 mins until a steady temperature was reached in the motors and the nut.

\section{RESULTS AND DISCUSSION}

\section{A. Feature extraction}

As mentioned in section II.A, the extraction of features from the signal is critical for the performance of the monitoring algorithm. For that reason the features set was chosen for likely sensitivity to degradation, and then tested for effectiveness. The correlation between these features was studied using PCA in order to establish a baseline that defines the normal behaviour of the system in absence of faults under different loading scenarios. As an additional benefit this procedure reduces significantly the amount of data analysed. During the training phase, using data acquired in absence of faults, these features

\section{Trapezoidal profile}

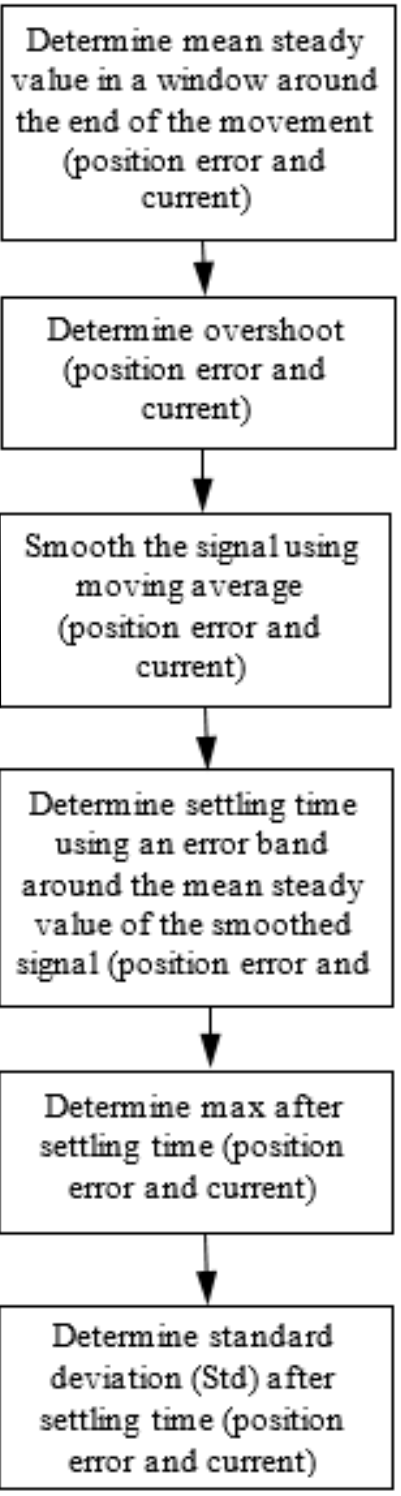

Sinusoidal profile

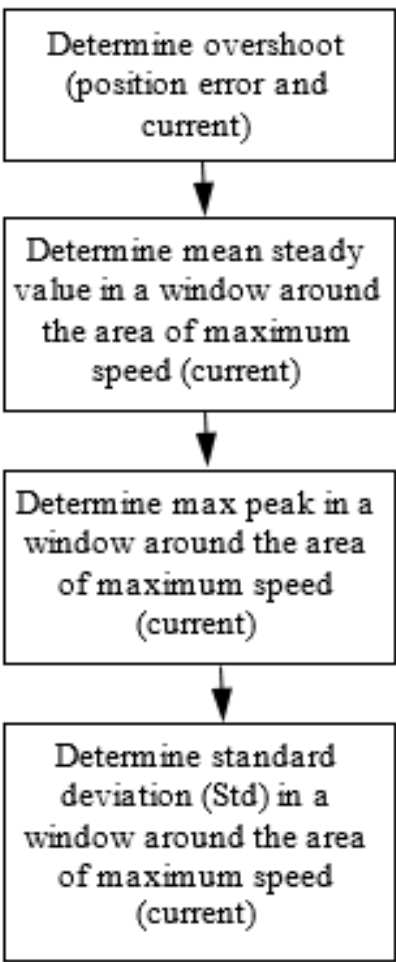

Fig. 8: Feature extraction procedure for trapezoidal and sinusoidal motion profiles were combined to capture the system dynamics under different operating scenarios. In subsequent tests, any deviations found in these features were examined for indication of the presence of faults in the system.

Due to the differences in the signals acquired for trapezoidal and sinusoidal motion profiles the sets of features extracted and the extraction method in each case are slightly different. These features were extracted from position error and current signals independently for the extension and retraction movement in each complete motion, and each motion type was analysed separately. Fig. 8 summarises the automated feature extraction procedure used in each case.

For the trapezoidal profile, the last 80 samples (corresponding to $3.2 \mathrm{~s}$ of measurements) from position error and current measurements of each movement were used to calculate the mean steady value. Both signals were filtered using a moving average with a window length of 20 and 15 data points respectively. This filtering process produces a smoothed signal eliminating random noise that allows for a more accurate estimation of the setting time (see Fig. 9). The settling time was established as the last data point in the filtered signal with an absolute value lower than $0.5 \mathrm{~mm}$ for position error and lower than $10 \%$ of the mean steady current for the current signal. These threshold values were set empirically after analysing the behaviour of the signals under different operating conditions. Finally the amplitude of the maximum deviation with respect to the mean steady value and the standard deviation between the settling time and the end of the movement was computed for both signals. An example of features captured can be seen in Fig. 9 a). Table I shows the final list of features extracted from both signals for the trapezoidal case and the ID number assigned to each of these features, which will be referred to in section IV.

In the case of the sinusoidal profile, due to the constant change in the speed set point, none of the signals ever reached a real "steady value". In the case of the position error, only the overshoot value was captured. For current measurements, in addition to the overshoot, a region around the maximum speed point with a speed variation lower than $10 \%$ was further analysed. The current signal was considered almost steady in this region, and the mean value, the amplitude of the maximum deviation from the mean and the standard deviation were extracted from this signal section. Table II shows the list of features extracted from both signals for the sinusoidal case.

\section{B. Analysis of extracted features}

\section{1) Normal operation}

In order to train the algorithm and establish a set of baseline values for the features extracted, the first dataset was acquired in normal conditions (absence of faults) for the trapezoidal and sinusoidal motion profiles under the three loading scenarios considered. Fig. 10 shows an example of some features (measurements 1,2,5,11,12 and 15 in Table I) extracted from 

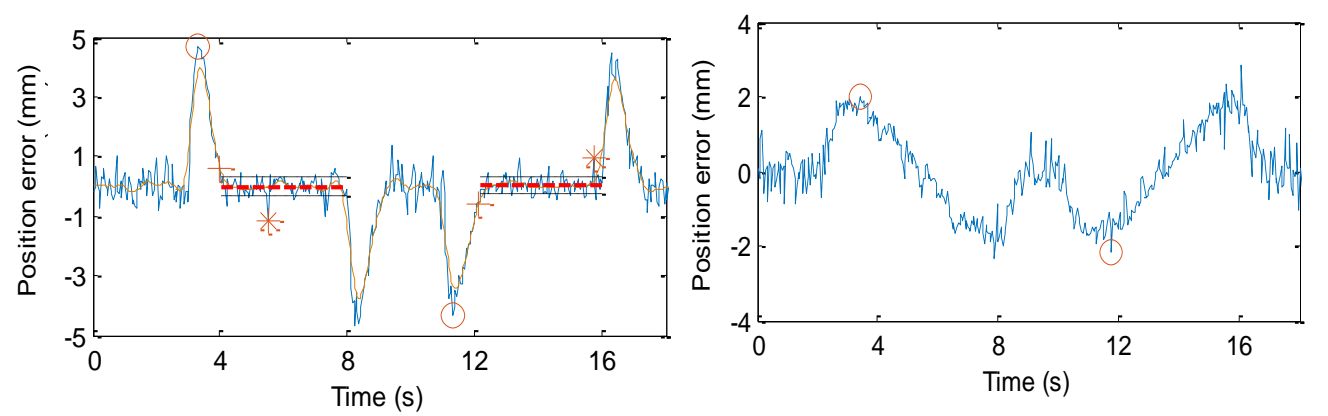

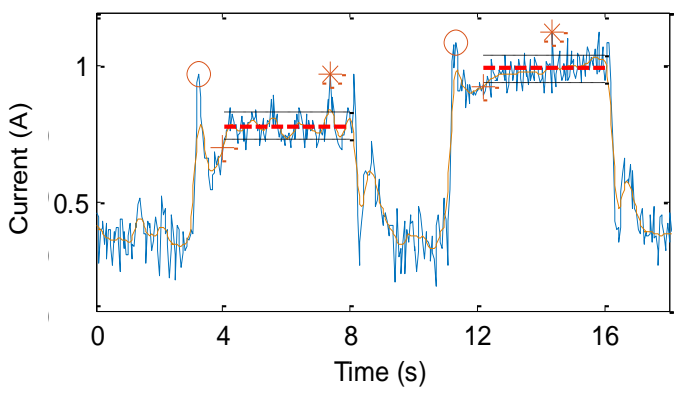

a)

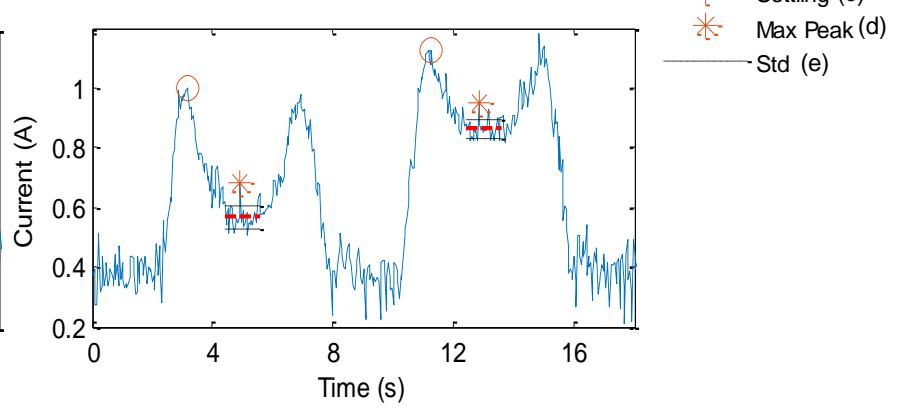

b)

Fig. 9: Example of features extracted for trapezoidal a) and sinusoidal b) motion

trapezoidal type extension movements for different loading conditions. These graphs show the variation in the features extracted due to noise, measurement error and variations in the operating conditions. Some of the features, especially those related to position measurements, show almost no variation for the different loading scenarios, as expected under closed loop control. However current measurements are hugely affected by the operating conditions. That is why it is necessary to produce a condition indicator that can characterise the health of the system under varying operating conditions for this application.

TABLE I:

FEATURES STRUCTURE FOR TRAPEZOIDAL PROFILE (FEATURES FROM II.A)

\begin{tabular}{|c|c|c|c|c|}
\hline Signal & Movement & Feature & $\begin{array}{l}\text { Feature } \\
\text { ID }\end{array}$ & $\begin{array}{l}\text { Measurement } \\
\text { ID }\end{array}$ \\
\hline \multirow{10}{*}{$\begin{array}{l}\text { Position } \\
\text { error }\end{array}$} & \multirow{5}{*}{ Extension } & Steady value & $\mathrm{a}$ & 1 \\
\hline & & Overshoot & $\mathrm{b}$ & 2 \\
\hline & & settling time & $\mathrm{c}$ & 3 \\
\hline & & Unexpected peaks & $\mathrm{d}$ & 4 \\
\hline & & Steady region Std. & $\mathrm{e}$ & 5 \\
\hline & \multirow{5}{*}{ Retraction } & Steady value & $\mathrm{a}$ & 6 \\
\hline & & Overshoot & $\mathrm{b}$ & 7 \\
\hline & & settling time & $\mathrm{c}$ & 8 \\
\hline & & Unexpected peaks & $\mathrm{d}$ & 9 \\
\hline & & Steady region Std. & e & 10 \\
\hline \multirow{10}{*}{ Current } & \multirow{5}{*}{ Extension } & Steady value & $\mathrm{a}$ & 11 \\
\hline & & Overshoot & $\mathrm{b}$ & 12 \\
\hline & & settling time & $\mathrm{c}$ & 13 \\
\hline & & Unexpected peaks & $\mathrm{d}$ & 14 \\
\hline & & Steady region Std. & e & 15 \\
\hline & \multirow{5}{*}{ Retraction } & Steady value & a & 16 \\
\hline & & Overshoot & $\mathrm{b}$ & 17 \\
\hline & & settling time & $\mathrm{c}$ & 18 \\
\hline & & Unexpected peaks & $\mathrm{d}$ & 19 \\
\hline & & Steady region Std. & $\mathrm{e}$ & 20 \\
\hline
\end{tabular}

The training data set was composed of 150 observations (50 for each loading condition tested) and 20 measurements were analysed following the procedure described in section II.B. The number of principal components to retain $a$ was chosen so that at least $80 \%$ of the variability in the data is retained. According to the analysis of the singular values the number of retained principal components was set to 2 . The probability density function of the $\mathrm{T}^{2}$ and $\mathrm{Q}$ indicators was estimated using KDE. The thresholds were calculated for a confidence bound of $90 \%$, obtaining values of 17.88 and 18.89 for $\mathrm{T}^{2}$ and $\mathrm{Q}$ respectively.

Fig. 11 (a) shows the values of both indicators against their respective thresholds for the three loading scenarios in absence of faults. This result shows the ability of PCA to provide information about the system condition for different operating conditions. Fig. 11 (b) shows the scores for the two main principal components, which is a measurement of the weight of each feature measured in each dimension of the principal component space.

TABLE II:

FEATURES STRUCTURE FOR SINUSOIDAL PROFILE (FEATURES FROM II.A)

\begin{tabular}{lllll}
\hline \hline Signal & Movement & Feature & $\begin{array}{l}\text { Feature } \\
\text { ID }\end{array}$ & $\begin{array}{l}\text { Measurement } \\
\text { ID }\end{array}$ \\
\hline $\begin{array}{l}\text { Position } \\
\text { error }\end{array}$ & Extension & Overshoot & $\mathrm{b}$ & 1 \\
& Retraction & Overshoot & $\mathrm{b}$ & 2 \\
\hline \multirow{4}{*}{ Current } & \multirow{3}{*}{ Extension } & Steady value & $\mathrm{a}$ & 3 \\
& & Overshoot & $\mathrm{b}$ & 4 \\
& & Unexpected peaks & $\mathrm{d}$ & 5 \\
& & Steady region Std. & $\mathrm{e}$ & 6 \\
& & Steady value & $\mathrm{a}$ & 7 \\
& \multirow{2}{*}{ Retraction } & Overshoot & $\mathrm{b}$ & 8 \\
& & Unexpected peaks & $\mathrm{d}$ & 9 \\
& & Steady region Std. & $\mathrm{e}$ & 10 \\
\hline \hline
\end{tabular}



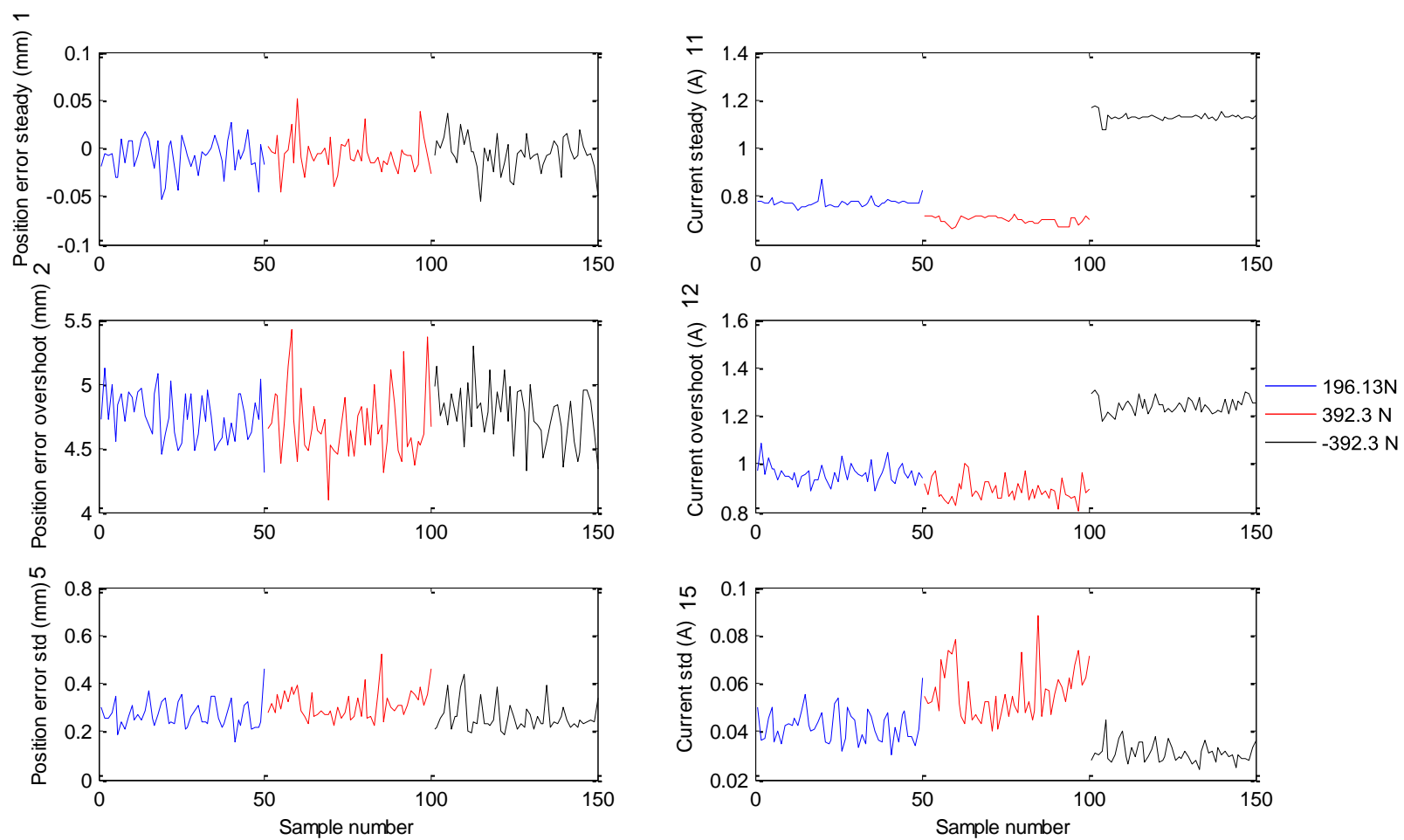

Fig. 10 Example of features from trapezoidal extension movement

The same analysis was undertaken for the sinusoidal profile dataset. In this case also two principal components were retained and the threshold values were set to 13.75 and 5.85 for the $\mathrm{T}^{2}$ and $\mathrm{Q}$ indicators respectively. Fig. 12 summarises the results obtained in this case.

\section{2) Lack of lubrication}

This case was studied in two stages with increasing severity. Fig. 13 (a) shows the condition indicators for this case for the trapezoidal profile, where the first 150 samples correspond to the first stage and the remaining 150 observations correspond
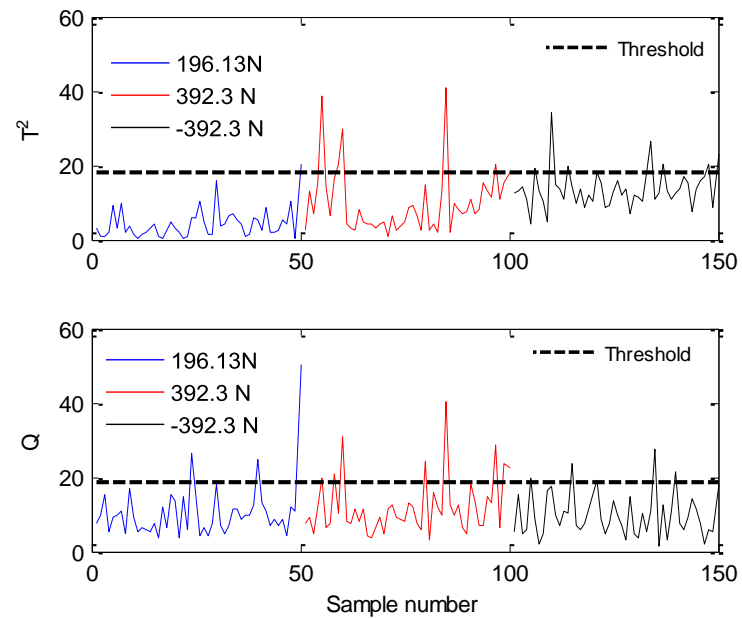

a) to the second stage. The detection rate for the $\mathrm{T}^{2}$ indicator was $48.6 \%$ and $64.7 \%$ for the 1 st and 2nd stages of the test respectively. The $\mathrm{Q}$ indicator showed a better performance with $88 \%$ and $100 \%$ detection rate for the $1^{\text {st }}$ and $2^{\text {nd }}$ stages. Different values of number of principal components (PCs) retained $a$ were tried to improve the performance of the $\mathrm{T}^{2}$ indicator, obtaining poorer results. As a consequence this parameter was kept to 2. The lack of detection of the $\mathrm{T}^{2}$ indicator is attributed to the fact that this particular fault tends to produce features located away from the retained PCs rather than changing the values of the features within the retained space.
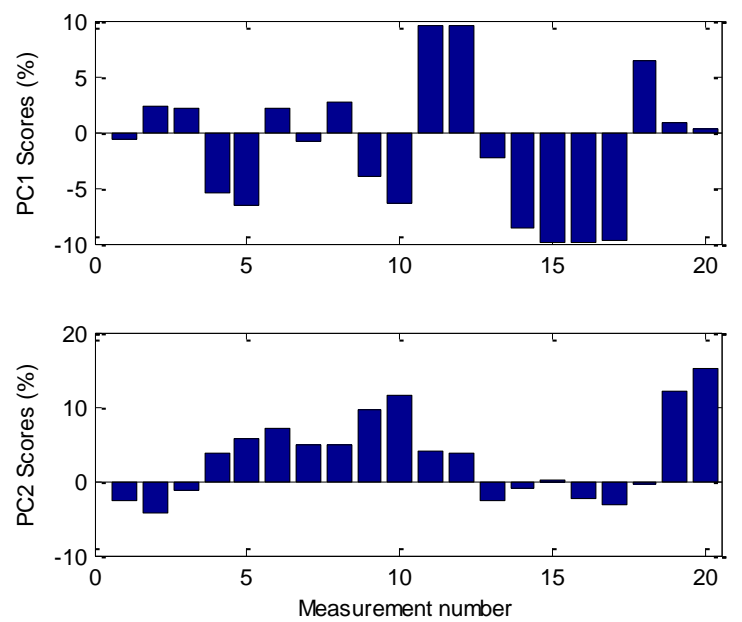

b)

Fig. 11 Condition indicators (a) and PC scores (b) for the trapezoidal training data set 

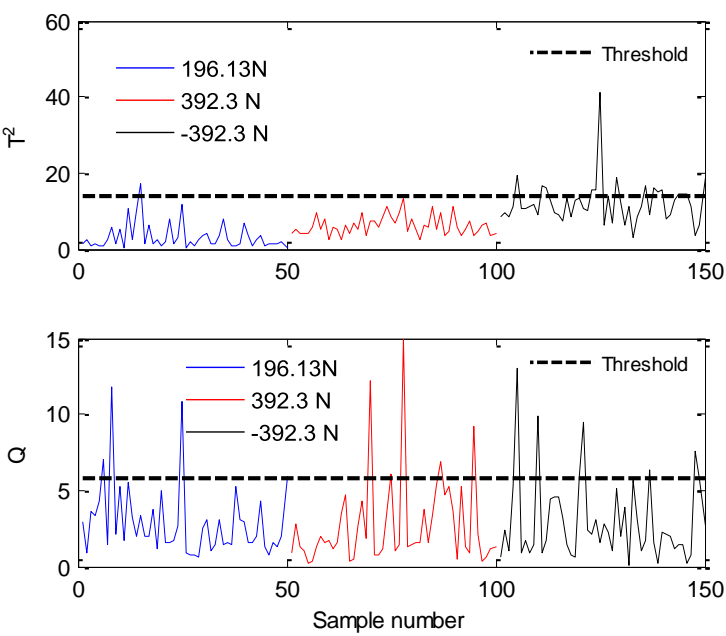

a)
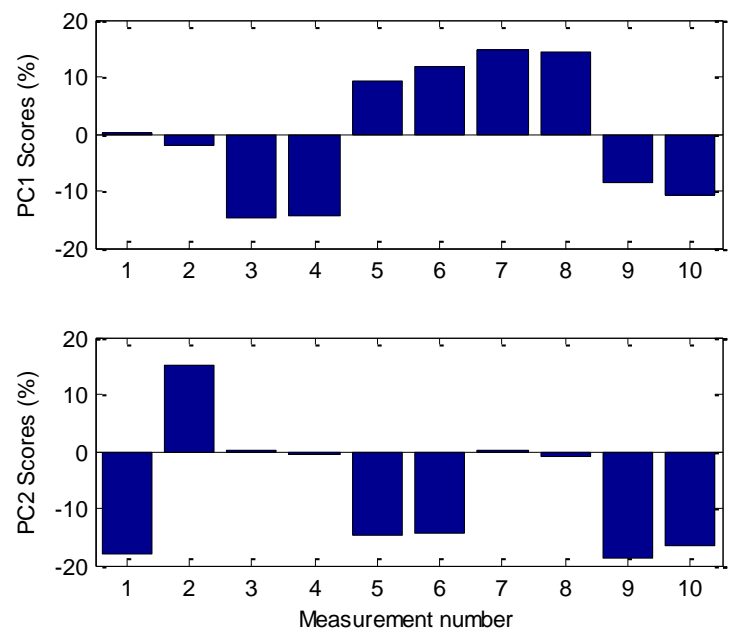

b)

Fig. 12 Condition indicators (a) and PC scores (b) for the sinusoidal training data set

Fig. 13 (b) shows an example the contribution of each measurement to each indicator at sample number 15 (first stage, $196.13 \mathrm{~N}$. The measurements with higher contributions in both indicators are 12 and 17, which correspond with the current overshoot during the extension and retraction movements respectively. Measurements 11 and 16 (steady value) have also a high contribution. This is attributed to the excess of current that is needed to overcome the increment in friction and the change produced in the dynamic properties of the system.

The same analysis was performed for the sinusoidal profile, obtaining the results shown in Fig. 14. In this case the detection rate for the $\mathrm{T}^{2}$ indicator was $29.3 \%$ and $22.7 \%$ for the $1^{\text {st }}$ and $2^{\text {nd }}$ degradation stages respectively, while for the $\mathrm{Q}$ indicator it was $90 \%$ and $99.3 \%$. The main measurements contributing to the $\mathrm{T}^{2}$ indicator at sample $79\left(1^{\text {st }}\right.$ stage, $\left.392.3 \mathrm{~N}\right)$ were 3 and 7 , corresponding again to the current steady value in each movement direction. For the Q indicator measurement 7 was especially relevant at this particular sample.
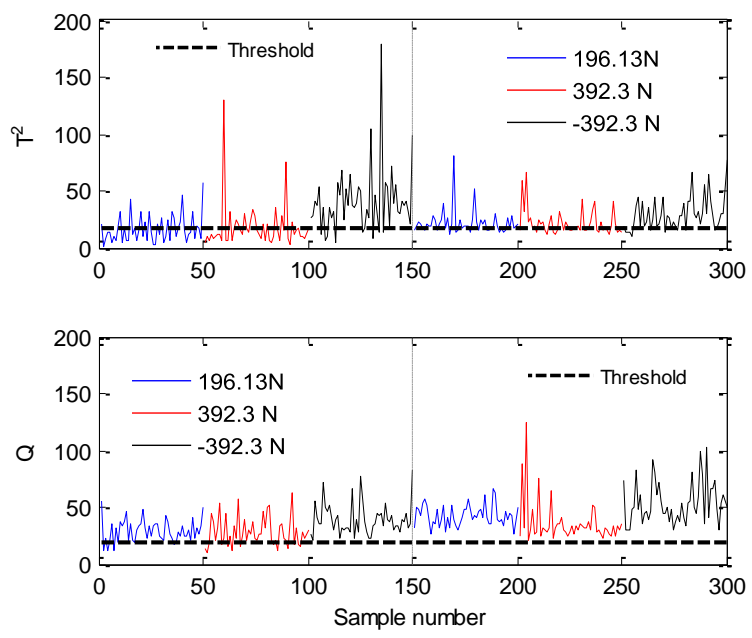

a)

\section{3) Spalling}

As explained in section III.B, spalling was introduced gradually in 8 stages. Despite the apparent severity of the fault, the system proved to be quite robust and continued working almost flawlessly even with defects of considerable size. Even in the last stages tested the momentary ball nut jamming did not occur in every single movement repetition, it only happened momentarily in some of the movements arbitrarily. During the tests it was observed that for the sinusoidal profile (where the maximum speed is higher than for the trapezoidal case) momentary jamming was quite frequent during the 7 th degradation stage. Fig. 15 and Fig. 16 show the results obtained for these tests. Detection rate results are presented in Table III.

The detection rate results summarised in Table III reveal that for the trapezoidal profile tests the number of threshold crossings was obviously high only in the $8^{\text {th }}$ stage, and moderate in the $6^{\text {th }}$ and $7^{\text {th }}$ stages. For the first 5 stages the results are quite
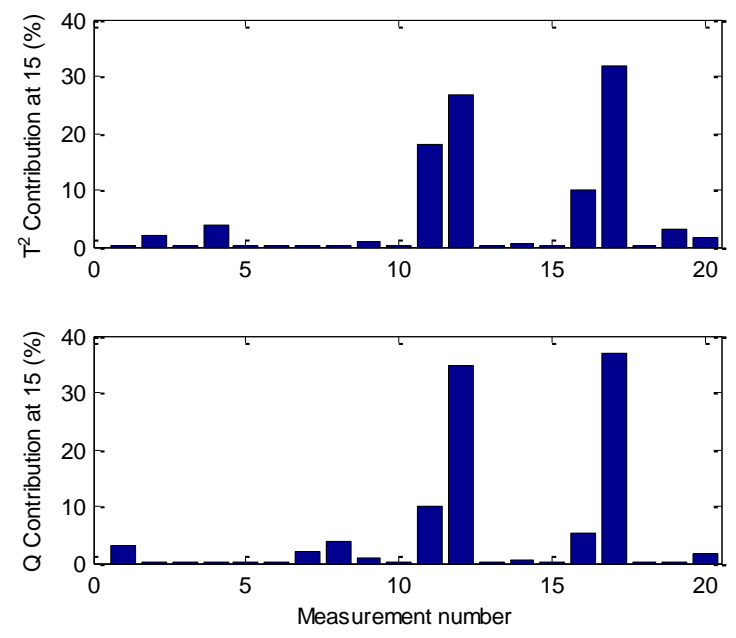

b)

Fig. 13 Condition indicators (a) and contributions at sample 15 (b) for lack of lubrication (trapezoidal) 

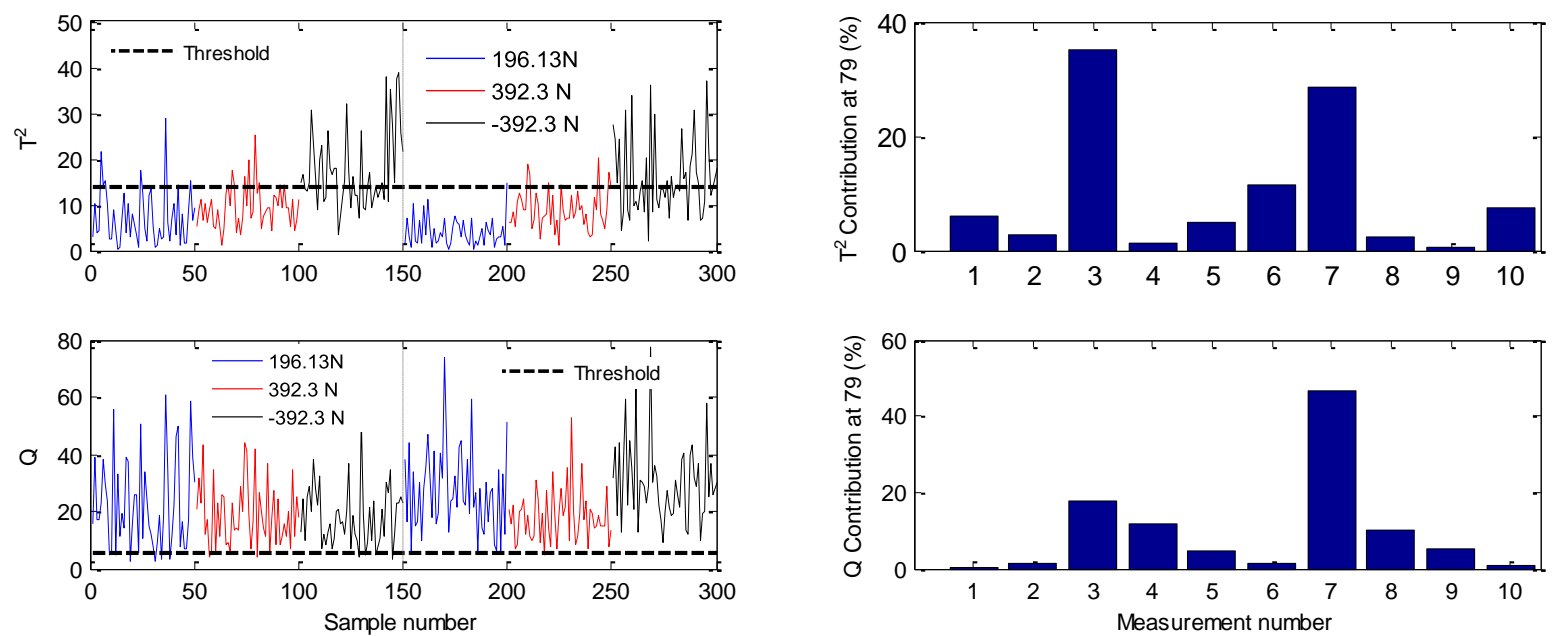

a)

b)

Fig. 14 Condition indicators (a) and contributions at sample 79 (b) for lack of lubrication (sinusoidal)
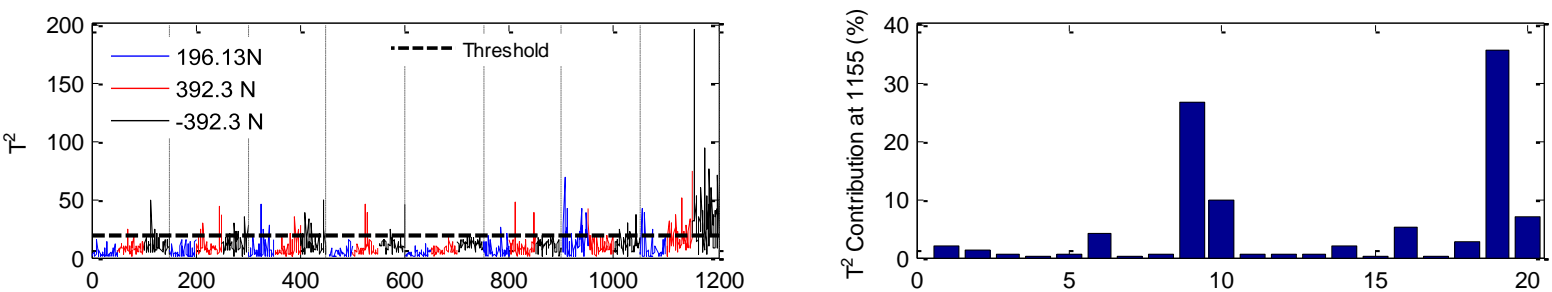

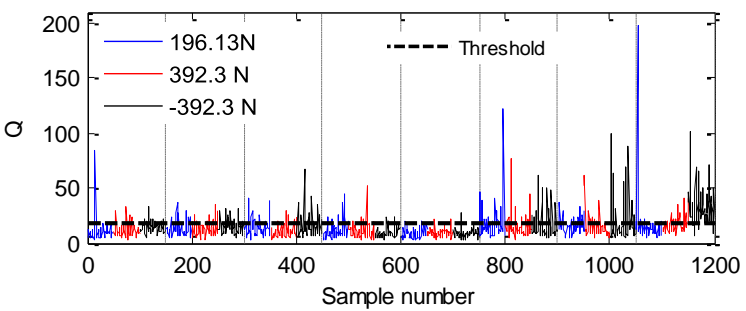

a)

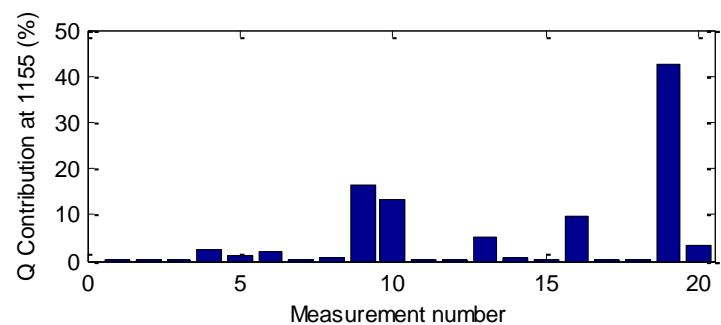

b)

Fig. 15 Condition indicators (a) and contributions at sample 1155 (b) for spalling (trapezoidal)
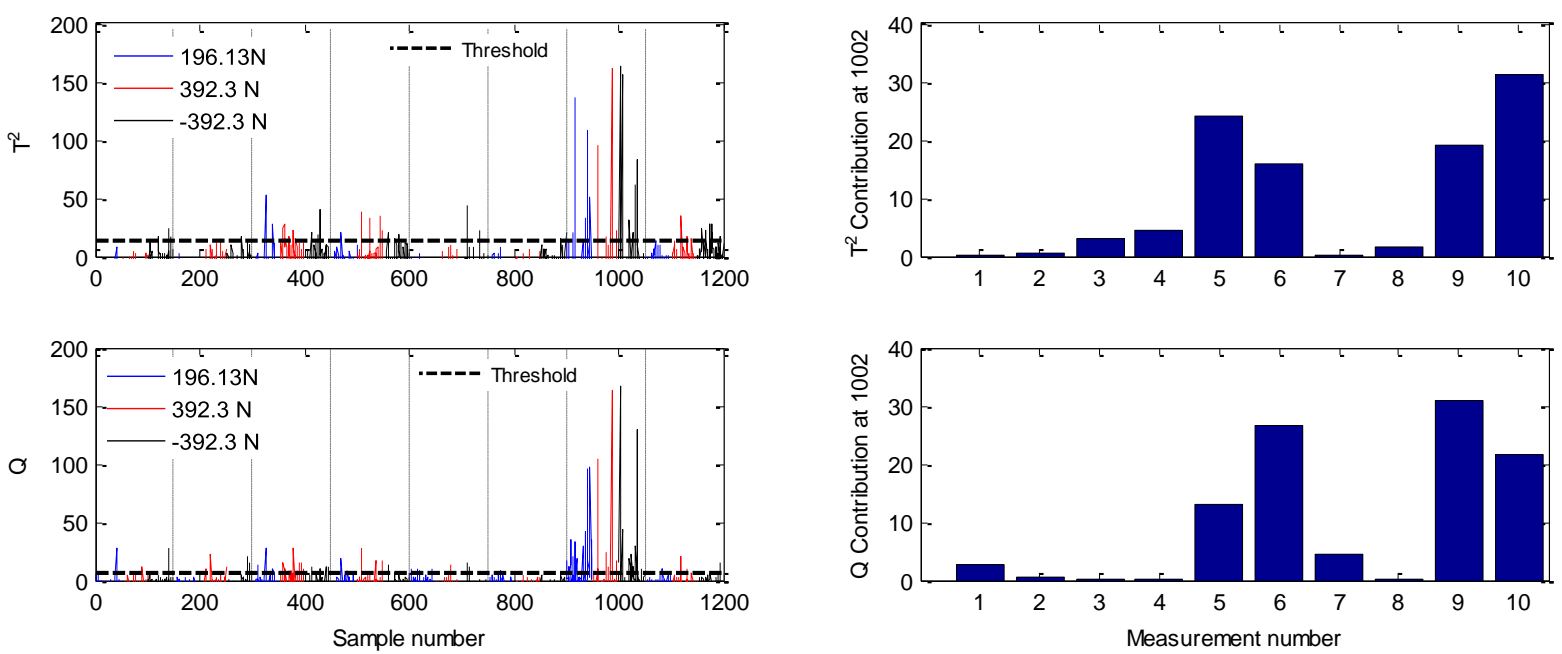

a)

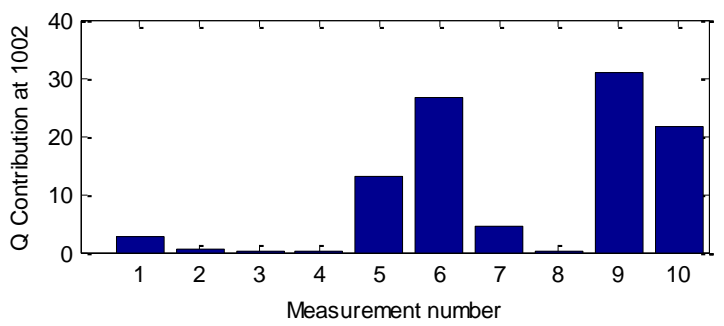

b)

Fig. 16 Condition indicators (a) and contributions at sample 1002 (b) for spalling (sinusoidal) 
TABLE III:

DETECTION RATES FOR SPALLING TESTS IN \%

\begin{tabular}{cllll}
\hline Degradation & \multicolumn{2}{c}{ Trapezoidal } & \multicolumn{2}{c}{ Sinusoidal } \\
stage & $\mathrm{T}^{2}$ & $\mathrm{Q}$ & $\mathrm{T}^{2}$ & $\mathrm{Q}$ \\
\hline 1 & 4.0 & 8.6 & 2.6 & 5.3 \\
2 & 7.3 & 21.3 & 1.3 & 4.0 \\
3 & 10.6 & 20.0 & 8.0 & 16.6 \\
4 & 3.3 & 11.3 & 5.3 & 5.3 \\
5 & 2.0 & 3.3 & 1.3 & 4.6 \\
6 & 6.6 & 27.3 & 1.3 & 1.3 \\
7 & 22.0 & 24.0 & 12.6 & 29.3 \\
8 & 37.3 & 45.3 & 9.3 & 5.3 \\
\hline \hline
\end{tabular}

similar to the training data set, showing that the algorithm was not able to detect the fault at these early stages. According to Fig. 15 (b) the measurements that contribute more to both indicators at sample $1155\left(8^{\text {th }}\right.$ stage under $\left.-392.3 \mathrm{~N}\right)$ are $9,10,19$ and 20 , which are related with the maximum deviations found during the steady movement region and the overall standard deviation in that same region of the two signals analysed, particularly for the retraction movement.

For the sinusoidal profile the indicators only gave a relatively high number of threshold crossings in the $7^{\text {th }}$ stage. These results are in line with the observations during the tests, where the actuator was operating in a normal way for the smaller defects tested. Ball nut jamming was only frequent for the sinusoidal profile during the $7^{\text {th }}$ stage. This is attributed to a combination of the high speed achieved during the sinusoidal movement and the sharpness of the defect edges in that particular stage. For the $8^{\text {th }}$ stage the defect size was bigger, but the edges of the defect were smoother. The three independent rows of balls make the system quite robust to this type of fault as the load is shared amongst many balls at different locations. According to Fig. 16 (b) the measurements that contribute more to both indicators at sample 1022 ( $7^{\text {th }}$ stage under $-392.3 \mathrm{~N}$ ) are $5,6,9$ and 10, which again are related with the maximum amplitude found during the steady movement region and the standard deviation in that same region of the current signal.
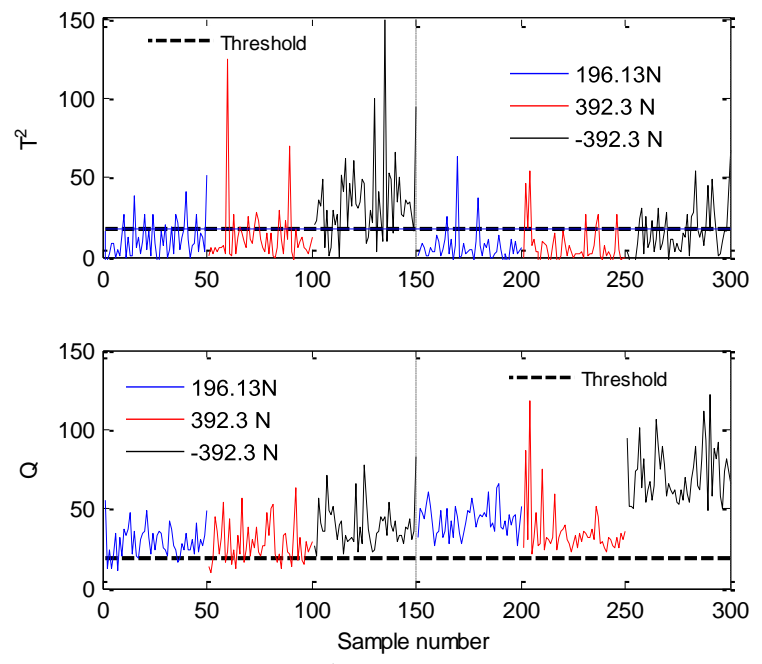

a)

\section{4) Backlash}

Backlash was introduced in two stages. Fig. 17 shows the results obtained for the trapezoidal motion profile. In this case the performance of the $\mathrm{T}^{2}$ indicator was relatively poor, giving a detection rate of $40.7 \%$ and $16 \%$ for stages 1 and 2 respectively, compared with the $88 \%$ and $100 \%$ detection rate given by the $\mathrm{Q}$ indicator in the same conditions. At sample 284 (second stage under $-392.3 \mathrm{~N}$ ) there are several variables contributing to the $\mathrm{T}^{2}$, which is an indication of its poor performance. The main contributions of the $\mathrm{Q}$ indicator at this data point are variables 5,10, 15 and 20, which are related with the standard deviation during the steady motion regions. This may be an indication of increment in the variability of the signals due to the effect of backlash.

Fig. 18 shows the results obtained for the sinusoidal motion profile. In this case the detection rates for the $\mathrm{T}^{2}$ indicator are $29.3 \%$ and $76 \%$ for the first and second degradation stages. Again the contribution of the $\mathrm{T}^{2}$ indicator at sample $210\left(2^{\text {nd }}\right.$ stage under $392.3 \mathrm{~N}$ ) is shared amongst many variables, which hinders the diagnosis. The $\mathrm{Q}$ indicator however shows much better detection rates $90 \%$ in the first stage and $100 \%$ in the second. Additionally the measurements contributing more to this indicator at sample 210 are 6 and 10 , measuring the standard deviation in the steady region of the current signals in both directions.

\section{CONCLUSION}

This paper presents a novel method for linear actuator monitoring based on extraction of key features from current and position measurements. These features are carefully selected in order to obtain a good representation of the system dynamics during transient and steady operation with a reduced data set. These features are combined using PCA to produce condition indicators that can be used for fault detection, as they are able to indicate changes in the system dynamics even under different
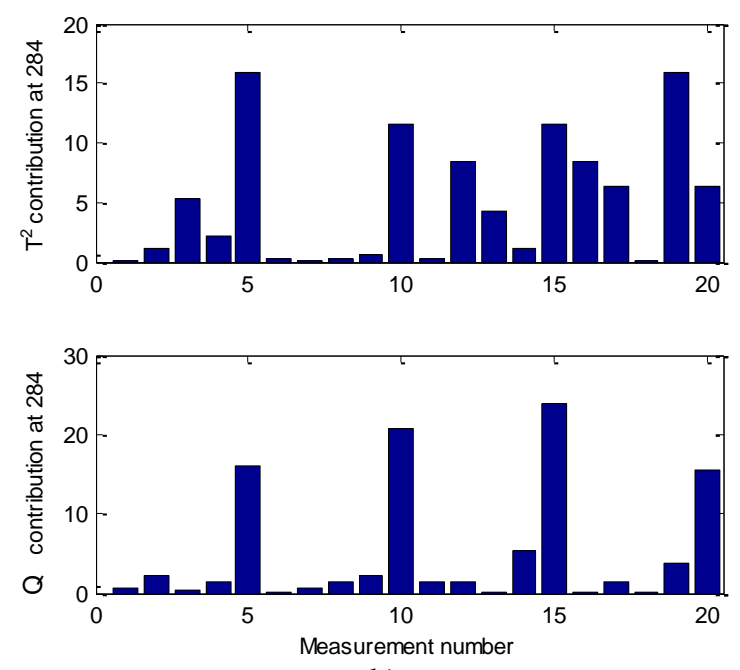

b)

Fig. 17 Condition indicators (a) and contributions at sample 284 (b) for backlash (trapezoidal) 

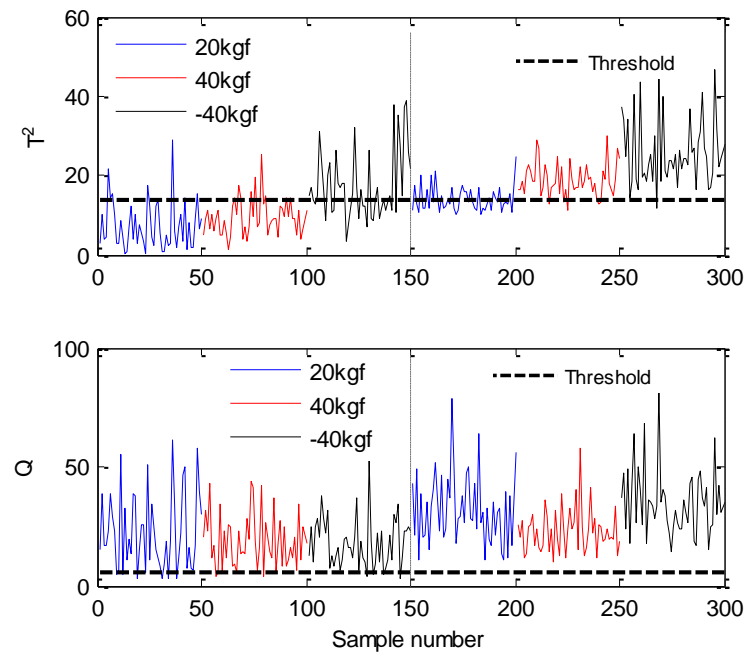

a)
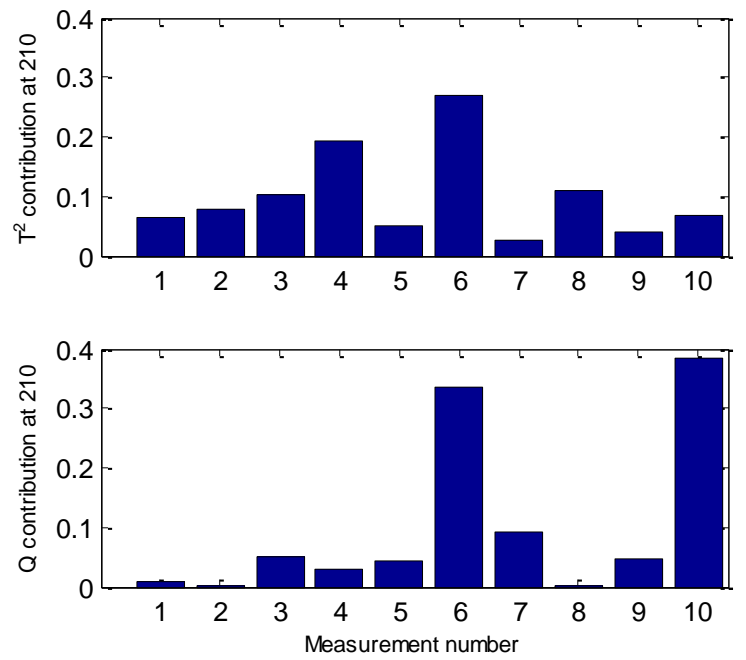

b)

Fig. 18 Condition indicators (a) and contributions at sample 210 (b) for backlash (sinusoidal)

operating conditions such as different loading scenarios. In addition, the capabilities of PCA to eliminate random variations in the data and capture systematic trends helped to reduce background noise in the signals.

The proposed approach was tested using experimental data acquired from a rig designed to operate under different loading conditions and allowing faults to be seeded. Data was acquired from position and current measurements under normal conditions, with lack of lubrication, spalling and with worn balls to simulate backlash. The sensors characteristics were selected to provide accurate and reliable measurements able to represent the characteristics of the system dynamics. Although the level of noise observed in the signals was relatively high, the PCA-based feature combination and dimensionality reduction provided consistent health indicators even under different loads.

The rig operation proved to be quite robust for these particular fault scenarios. It continued operating even when the faults introduced were visually quite severe. The results obtained show that the algorithm was able to detect the faults introduced, although the detection of these faults in the very early stages of degradation was not always possible due to the mentioned robustness of the system.

The results obtained showed that the proposed feature extraction and analysis approach proposed was able to detect effectively lack of lubrication and backlash under different motion and loading conditions. Particularly the Q indicator showed a low false alarm rate, and the $\mathrm{Q}$ contribution plots provided valuable information for fault diagnosis. The $\mathrm{T}^{2}$ indicator on the other hand typically showed low detection rates, despite the efforts made in tuning the algorithm parameters to improve its performance. Both indicators failed to detect spalling in the first stages of degradation, but during the tests it was observed that the mechanism performance and behaviour was not very different from what was observed in absence of faults. When the defect introduced was big enough to affect the behaviour of the mechanism considerably the algorithm was able to detect the fault and provide information about its origin. The use of multivariate analysis was crucial to simplify the fault detection and diagnosis problem involving several variables and changing operation.

The superior performance of the $\mathrm{Q}$ indicator over $\mathrm{T}^{2}$ has also been observed in other investigations. Generally it is attributed to the fact that most faults tend to produce features located away from the retained principal components rather than changing the values of the features within the retained space. Although different tests were carried out retaining a higher number of principal components, the best overall detection performance was found setting this parameter to 2 .

The analysis of the contribution of each measurement to the indicators' value provided very useful information about the origin of the source of the threshold crossing. Some of the selected features produced significantly high scores during the training phase, as they contain most of the data variability in such conditions. However, some of the features with lower scores were determinant in the detection of some faults, proving the need to include such features to provide accurate diagnosis information. This information could be used, not only to detect the fault automatically, but also to guide subsequent inspection to confirm the origin and extent of the fault, which some industries may require before commissioning replacement or repair. In order to improve the performance of the proposed approach in future investigations the exploration of indicator combination and the comparison of measurements with historic data and previous operations for building confidence in diagnosis is suggested. 


\section{REFERENCES}

[1] The National Transportation Safety Board, "Aviation Accident Report - Loss of Control and Impact with Pacific Ocean, Alaska Airlines Flight 261, McDonnell Douglas MD-83, N963AS, about 2.7 miles north of Anacapa Island, California." [Online]. Available: http://www.ntsb.gov/investigations/accidentreports/pages/AAR0201 .aspx. [Accessed: 15-Nov-2016].

[2] E. Balaban, A. Saxena, P. Bansal, K. F. Goebel, P. Stoelting, and S Curran, "A diagnostic approach for electro-mechanical actuators in aerospace systems," IEEE Aerosp. Conf. Proc., 2009.

[3] P. Phillips, D. Diston, and A. Starr, "Perspectives on the commercial development of landing gear health monitoring systems," Transp. Res. Part C Emerg. Technol., vol. 19, no. 6, pp. 1339-1352, 2011.

[4] P. Jänker, F. Claeyssen, B. Grohmann, M. Christmann, T. Lorkowski, R. LeLetty, O. Sosniki, and A. Pages, "New Actuators for Aircraft and Space Applications," in ACTUATOR 2008, 11th International Conference on New Actuators, 2008.

[5] P. Phillips and D. Diston, "A knowledge driven approach to aerospace condition monitoring," Knowledge-Based Syst., vol. 24, no. 6, pp. 915-927, Aug. 2011.

[6] S. C. Jensen, G. D. Jenney, and D. Dawson, "Flight test experience with an electromechanical actuator on theF-18 Systems Research Aircraft," 19th DASC. 19th Digit. Avion. Syst. Conf. Proc. (Cat. No.00CH37126), vol. 1, pp. 1-11, 2000.

[7] G. Swerdon, M. J. Watson, S. Bharadwaj, C. S. Byington, M. Smith, K. Goebel, and E. Balaban, "A Systems Engineering approach to Electro-Mechanical Actuator diagnostic and prognostic development," in Failure Prevention: Implementation, Success Stories and Lessons Learned - Proceedings of the 2009 Conference of the Society for Machinery Failure Prevention Technology, 2009.

C. S. Byington, M. Watson, D. Edwards, and P. Stoelting, "A model-based approach to prognostics and health management for flight control actuators," in IEEE Aerospace Conference Proceedings, 2004, vol. 6, pp. 3551-3562.

[9] L. U. Gökdere, S. L. Chiu, K. J. Keller, and J. Vian, "Lifetime control of electromechanical actuators," in IEEE Aerospace Conference Proceedings, 2005, vol. 2005.

[10] M. J. Smith, C. S. Byington, M. J. Watson, S. Bharadwaj, G. Swerdon, K. Goebel, and E. Balaban, "Experimental and analytical development of health management for electro-mechanical actuators," IEEE Aerosp. Conf. Proc., 2009.

[11] E. Balaban, A. Saxena, K. Goebel, C. S. Byington, M. Watson, S. Bharadwaj, and M. Smith, " Experimental Data Collection and Modeling for Nominal and Fault Conditions on ElectroMechanical Actuators," in Annual Conference of the Prognostics and Health Management Society , 2009.

[12] R. Dixon, N. Gifford, C. Sewell, and M. C. Spalton, "Reliable electrical actuation systems," IEE Colloq., no. 180, pp. 23-38, 1999.

[13] R. Dixon and A. W. Pike, "Application of condition monitoring to an electromechanical actuator: A parameter estimation based approach," Comput. Control Eng. J., vol. 13, no. 2, pp. 71-81, 2002.

[14] R. Dixon and A. W. Pike, "Improving the control and reliability of an electromechanical actuator," in IFAC Proceedings Volumes (IFAC-PapersOnline), 2005, vol. 16, pp. 329-334.

[15] R. Dixon, "Observer-based FDIA: application to an electromechanical positioning system," Control Eng. Pract., vol. 12, no. 9, pp. 1113-1125, Sep. 2004.

[16] A. J. Chirico III, J. R. Kolodziej, and L. Hall, "A data driven frequency based feature extraction and classification method for ema fault detection and isolation," in ASME 2012 5th Annual Dynamic Systems and Control Conference Joint with the JSME 2012 11th Motion and Vibration Conference, DSCC 2012-MOVIC 2012, 2012, vol. 2, pp. 751-760.

[17] A. J. Chirico III and J. R. Kolodziej, "A data-driven methodology for fault detection in electromechanical actuators," J. Dyn. Syst. Meas. Control. Trans. ASME, vol. 136, no. 4, 2014.

[18] A. Chirico and J. R. Kolodziej, "Fault Detection and Isolation for
Electro-Mechanical Actuators Using a Data-Driven Bayesian Classification,” pp. 494-502, Oct. 2012.

[19] S. Nandi, H. A. Toliyat, and X. Li, "Condition monitoring and fault diagnosis of electrical motors - A review," IEEE Trans. Energy Convers., vol. 20, no. 4, pp. 719-729, 2005.

[20] C. Ruiz-Carcel and A. Starr, "Data set for 'Data-based Detection and Diagnosis of Faults in Linear Actuators,'" [Online]. Available: https://doi.org/10.17862/cranfield.rd.5097649 [Accessed: 28-Feb2018].

[21] C. Ruiz-Carcel and A. Starr, "Development of a Novel Condition Monitoring Tool for Linear Actuators," in The Twelfth International Conference on Condition Monitoring and Machinery Failure Prevention Technologies, 2015.

[22] L. Xu, J. Chen, Z. Cao, W. Zhang, R. Xie, X. Liu, and J. Hu, "Identification of Oil-Water Flow Patterns in a Vertical Well Using a Dual-Ring Conductance Probe Array," IEEE Trans. Instrum. Meas., vol. 65, no. 5, pp. 1249-1258, 2016.

[23] A. Malhi and R. X. Gao, "PCA-based feature selection scheme for machine defect classification," IEEE Trans. Instrum. Meas., vol. 53, no. 6, pp. 1517-1525, 2004.

[24] L. H. Chiang and E. L. R. and R. D. Braatz, Fault detection and diagnosis in industrial systems, vol. 1. London UK: Springer, 2000.

[25] P.-E. P. Odiowei and Y. Cao, "Nonlinear dynamic process monitoring using canonical variate analysis and kernel density estimations," Comput. Aided Chem. Eng., vol. 27, no. C, pp. 15571562, 2009.

[26] C. Ruiz-Cárcel, Y. Cao, D. Mba, L. Lao, and R. T. Samuel, "Statistical process monitoring of a multiphase flow facility," Control Eng. Pract., vol. 42, pp. 74-88, 2015.

\section{ACKNOWLEDGMENTS}

The authors gratefully acknowledge the support of the Engineering and Physical Sciences Research Council (EPSRC) Centre for Through-life Engineering Services, Rolls-Royce, BAE Systems, Bombardier Transportation, Babcock and the Ministry of Defence. 
2018-03-27

\section{Data-based detection and diagnosis of faults in linear actuators}

Ruiz-Carcel, Cristobal

IEEE

Cristobal Ruiz-Carcel and Andrew Starr. Data-based detection and diagnosis of faults in linear actuators. IEEE Transactions on Instrumentation and Measurement, Volume 67, Issue 9, September 2018, pp2035-2047

https://doi.org/10.1109/TIM.2018.2814067

Downloaded from Cranfield Library Services E-Repository 